\title{
Quasielastic Scattering from Relativistic Bound Nucleons: Transverse-Longitudinal Response
}

\author{
J. M. Udias, ${ }^{1,2}$ J. A. Caballero, ${ }^{1,3}$ E. Moya de Guerra, ${ }^{1}$ J. E. Amaro, ${ }^{4}$ and T. W. Donnelly ${ }^{5}$ \\ ${ }^{1}$ Instituto de Estructura de la Materia, CSIC Serrano 123, E-28006 Madrid, Spain \\ ${ }^{2}$ Departamento de Física Atómica, Molecular y Nuclear, Universidad Complutense de Madrid, E-28040 Madrid, Spain \\ ${ }^{3}$ Departamento de Física Atómica, Molecular y Nuclear, Universidad de Sevilla, E-41080 Sevilla, Spain \\ ${ }^{4}$ Departamento de Física Moderna, Universidad de Granada, E-18071 Granada, Spain \\ ${ }^{5}$ Center for Theoretical Physics, Laboratory for Nuclear Science and Department of Physics, Massachusetts Institute of Technology, \\ Cambridge, Massachusetts 02139
}

(Received 26 April 1999)

\begin{abstract}
Predictions for electron induced proton knockout from $p_{1 / 2}$ and $p_{3 / 2}$ shells in ${ }^{16} \mathrm{O}$ are presented using various approximations for the relativistic nucleonic current. Results for differential cross section, transverse-longitudinal response $\left(R_{\mathrm{TL}}\right)$, and left-right asymmetry $A_{\mathrm{TL}}$ are compared at $\left|Q^{2}\right|=0.8(\mathrm{GeV} / c)^{2}$. We show that there are important dynamical and kinematical relativistic effects which can be tested by experiment.
\end{abstract}

PACS numbers: 25.30.Fj, 21.60.Cs, 24.10.-i, 25.30.Rw

The exclusive $\left(e, e^{\prime} p\right)$ coincidence measurement allows the determination of the missing energy $\left(E_{m}\right)$ and missing momentum ( $p_{m}$, the momentum of the recoiling nucleus) in the reaction, and information on the energies, momentum distributions, and spectroscopic factors of bound nucleons is provided [1,2]. Until recently low- $E_{m}$ data were concentrated at $p_{m} \leq 300 \mathrm{MeV} / c$. Now higher $p_{m}$ regions are being probed at small $E_{m}$ under quasielastic conditions [3], yielding new information of high momentum components of bound nucleons in a regime (Bjorken $x \simeq 1$ ) where two-body currents can be safely neglected [4,5]. Most theoretical work on $\left(e, e^{\prime} p\right)$ has been based on nonrelativistic approximations to the nucleon current, namely the standard distorted-wave impulse approximation (DWIA) [1]. DWIA data analyses [2] have met two major difficulties: (a) The spectroscopic factors extracted from low- $p_{m}\left(p_{m}<300 \mathrm{MeV} / c\right)$ data are too small (for instance, $S_{\alpha} \simeq 0.5$ for $3 s_{1 / 2}$ and $2 d_{5 / 2}$ orbits in ${ }^{208} \mathrm{~Pb}[2,6]$ ), while theories on short-range correlations [7] predict at most a $30 \%$ reduction of mean-field occupations $\left(S_{\alpha}>\right.$ 0.7 ) for levels just below the Fermi level. (b) DWIA calculations compatible with the low- $p_{m}$ data predict much smaller cross sections at high $p_{m}\left(p_{m}>300 \mathrm{MeV} / c\right)$ than those experimentally observed [6]. Short-range correlations are expected to increase the high-momentum components, but their effect is negligible [8] at the small missing energies of these high- $p_{m}$ data [6], and long-range correlations have been introduced to explain the high- $p_{m}$ data within the nonrelativistic formalism $[4,6]$.

In recent years the relativistic mean-field approximation has been sucessfully used for the analyses of both low- $p_{m}$ [9-13] and high- $p_{m}$ [14] data. In the relativistic distorted-wave impulse approximation (RDWIA), the nucleon current

$$
J_{N}^{\mu}(\omega, \vec{q})=\int d \vec{p} \bar{\psi}_{F}(\vec{p}+\vec{q}) \hat{j}_{N}^{\mu}(\omega, \vec{q}) \psi_{B}(\vec{p})
$$

is calculated with relativistic $\psi_{B}\left(\psi_{F}\right)$ wave function for the initial bound (final outgoing) nucleon. $\hat{J}_{N}^{\mu}$ is the relativistic nucleon current operator of $c c 1$ or $c c 2$ forms as in [15]. For $\psi_{B}$ we use Dirac-Hartree solutions from relativistic Lagrangians with scalar and vector meson terms [16]. For $\psi_{F}$ we compute a solution of the Dirac equation with scalar-vector (S-V) global optical potentials [17]. The only fitted parameter is the spectroscopic factor [10-13]. These RDWIA spectroscopic factors are larger than the DWIA ones [11-13], and are valid both for low- and high- $p_{m}$ data [14].

Exploratory studies within the relativistic plane wave impulse approximation (RPWIA), where the interaction in the final state (FSI) between the ejected nucleon and the residual nucleus is neglected, showed [18-20] that the transverse-longitudinal response $\left(R_{\mathrm{TL}}\right)$ is very sensitive to the negative-energy components of the relativistic bound nucleon wave function. In a recent work [19] we have also shown that, for the $j=l \pm 1 / 2$ spin-orbit partners of a given shell, this sensitivity is much larger for the $j=l-1 / 2$ than for the $j=l+1 / 2$ case. In this work we observe for the first time this striking feature in a more realistic description of the $\left(e, e^{\prime} p\right)$ reaction including relativistic FSI.

A certain degree of controversy surrounds the TL response measured in exclusive quasielastic electron scattering from the least bound protons in ${ }^{16} \mathrm{O}$ : in some cases [21] large deviations from standard DWIA calculations appear, while in others [9] the data are close to the calculations. New data on $R_{\mathrm{TL}}$ for proton knockout from the $1 p_{1 / 2}$ and $1 p_{3 / 2}$ orbits of ${ }^{16} \mathrm{O}$ are available from Jefferson Laboratory (TJNAF) experiment 89-003 [3] at $\left|Q^{2}\right| \cong 0.8(\mathrm{GeV} / c)^{2}$. In this work we show that there are important kinematical and dynamical relativistic effects for this case.

We compute $\psi_{B}$ with the parameters of the set NL-SH [22]. For $\psi_{F}$ we solve the Dirac equation with the energy-dependent, $A$-independent, potentials derived by Clark et al. [17] for ${ }^{16} \mathrm{O}$. Using other relativistic potentials available in the literature does not alter our conclusions. 
The kinematical setup follows the TJNAF proposal [3]: beam energy $2445 \mathrm{MeV},|\vec{q}| \simeq 1 \mathrm{GeV} / c, \omega \simeq 439 \mathrm{MeV}$, corresponding to quasielastic conditions $\left(\omega \simeq\left|Q^{2}\right| / 2 M\right)$, and proton kinetic energy of about $427 \mathrm{MeV}$.

We divide the differences between this fully relativistic approach and the standard nonrelativistic one into two categories: (i) Effects due to the relativistic 4-vector current operator, compared to the nonrelativistic one which involves $\vec{p} / M$ expansions. We call these effects kinematical because they are independent of the dynamics introduced by the nuclear interaction. (ii) Effects due to the differences between relativistic and nonrelativistic nucleon wave functions, which depend on the 4-spinor structure and importantly on the potentials used in the respective Dirac and Schrödinger equations. We call these effects dynamical. We recall that in nonrelativistic approaches the one-body current operator is expanded in a basis of free-nucleon plane waves, which amounts to a truncation of the nucleon propagator that ignores negative-energy solutions of the free Dirac equation.

One may identify two types of relativistic dynamical effects: (a) Effects coming from the difference between the upper components of $\psi_{F}, \psi_{B}$ and the solutions of the Schrödinger equation. Assuming equivalent central and spin-orbit potentials, this difference stems from the well-known Darwin term. If the relativistic dynamics is known, one can deduce the Darwin term and construct an equivalent bispinor wave function $\chi$ to include its effect in the nonrelativistic nucleon current, thus removing this source of difference between relativistic and nonrelativistic results [12,23]. This is done, for instance, in Ref. [24]. The influence of this term on $\left(e, e^{\prime} p\right)$ observables has been demonstrated in several works $[11,12,25,26]$. It appears to be the main dynamical relativistic effect in the cross section in the low- $p_{m}$ region $[11,12]$, and is important for the correct determination of the spectroscopic factor from low- $p_{m}$ data. Its omission reduces the spectroscopic factor by $15 \%-20 \%$. We included it in all calculations presented here. (b) The other dynamical effect is due to the negative-energy components of the relativistic wave functions. Starting from Schrödinger-like solutions $\chi$ one may at best construct properly normalized four-spinors of the form

$$
\psi=\frac{1}{\sqrt{N}}\left[\chi(\vec{p}), \frac{\vec{\sigma} \cdot \vec{p}}{\hat{E}+M} \chi(\vec{p})\right]
$$

to calculate the relativistic nucleon current. This spinor lacks the dynamical enhancement due to the S-V potentials of the lower component of the Dirac solution. This dynamical enhancement is present in the relativistic $\psi_{B}, \psi_{F}$ solutions and influences $\left(e, e^{\prime} p\right)$ observables in the high- $p_{m}$ regions $[12,18]$.

Figure 1 shows the differential cross section, $R_{\mathrm{TL}}$ response, and TL asymmetry $\left(A_{\mathrm{TL}}\right)$ for $p_{1 / 2}$ (top panels) and $p_{3 / 2}$ (bottom panels). $R_{\mathrm{TL}}$ and $A_{\mathrm{TL}}$ are obtained from the difference of cross sections measured at $\phi_{F}=0^{\circ}$ and $\phi_{F}=180^{\circ}$ with the other variables $\left(\omega, Q^{2}, E_{m}, p_{m}\right)$ held constant, where $\phi_{F}$ is the azimuthal angle of the scattered proton (we follow the same convention as in [3]). Relativistic calculations using the $c c 1$ and $c c 2$ current operators are shown by solid and dotted lines, respectively. The Coulomb gauge has been used throughout. The use of an optical potential to account for inelastic reaction channels breaks Gauge invariance in DWIA. We estimated the uncertainty associated with the choice of gauge by comparing with results in the Landau gauge. For the fully relativistic results at the kinematics of this work, the difference in going from Coulomb to Landau Gauge is very small, much smaller than the difference between $c c 1$ and $c c 2$ results shown in the figures. We use a spectroscopic factor $S_{\alpha}=0.7$ as obtained for ${ }^{208} \mathrm{~Pb}$ [11], which in RDWIA also matches the low- $p_{m}$ data $[9,27]$ for the shells discussed here; the spectroscopic factor simply scales down the curves for differential cross sections and $R_{\mathrm{TL}}$ while leaving $A_{\mathrm{TL}}$ unchanged.

The role of the negative-energy components can be seen in Fig. 1. The short-dashed lines show the results obtained with the $c c 1$ current operator when the negative-energy components are projected out, i.e., the nucleon current is calculated as

$$
J_{\text {proj }}^{\mu}(\omega, \vec{q})=\int d \vec{p} \bar{\psi}_{F}^{(+)}(\vec{p}+\vec{q}) \hat{J}^{\mu}(\omega, \vec{q}) \psi_{B}^{(+)}(\vec{p}),
$$

where $\psi_{B}^{(+)}\left(\psi_{F}^{(+)}\right)$is the positive-energy component of $\psi_{B} \quad\left(\psi_{F}\right)$, i.e., $\psi_{B}^{(+)}(\vec{p})=\Lambda_{(+)}(\vec{p}) \psi_{B}(\vec{p}), \quad \Lambda_{(+)}(\vec{p})=$ $(M+\bar{p}) / 2 M$, with $\quad \bar{p}_{\mu}=\left(\sqrt{\vec{p}^{2}+M^{2}}, \vec{p}\right) \quad$ (similarly for $\left.\psi_{F}^{(+)}\right)$. The difference between the solid ( $c c 1$ full) and short dashed lines ( $c c 1$ projected) is due to the dynamical enhancement of the lower components which is contained in the current of Eq. (1), but not in Eq. (3). This effect is more visible than that introduced by the theoretical uncertainty due to the choice of $c c 1$ (solid line) or $c c 2$ (dotted line) operators. It is important to realize that the positive-energy projectors inserted in Eq. (3) depend on the integration variable $\vec{p}$. One may attempt to neglect this dependence by using projection operators corresponding to asymptotic values of the momenta, i.e., projectors acting on $\psi_{F}$ and $\psi_{B}$, respectively, with $P_{F}^{\mu}=\left(E_{F}, \vec{p}_{F}\right), P_{F}^{\mu}-\bar{Q}^{\mu}$ the asymptotic four-momentum of the outgoing and bound nucleon, respectively, with $\bar{Q}_{\mu}=\left[E_{F}-\sqrt{\left(\vec{p}_{F}-\vec{q}\right)^{2}+M^{2}}, \vec{q}\right]$. We refer to this approach as asymptotic projection $\left(J_{\mathrm{as}}\right)$. The corresponding results are shown by long dashed lines in Fig. 1. They are obtained with the $c c 1$ operator and are very similar for $c c 2$.

All the differential cross sections for $\left|p_{m}\right|<$ $300 \mathrm{MeV} / c$ are similar, but for $\left|p_{m}\right|>300 \mathrm{MeV} / c$ they show a substantial dependence on the negative-energy components for either the $p_{1 / 2}$ or $p_{3 / 2}$ shells. The cross sections obtained with $J_{\text {proj }}$ are more symmetrical around $p_{m}=0$ than the RDWIA results. Therefore, the effect of removing the negative-energy components shows up more in $R_{\mathrm{TL}}$ and $A_{\mathrm{TL}}$ (see middle and right-hand panels of Fig. 1). The dependence on the dynamical 

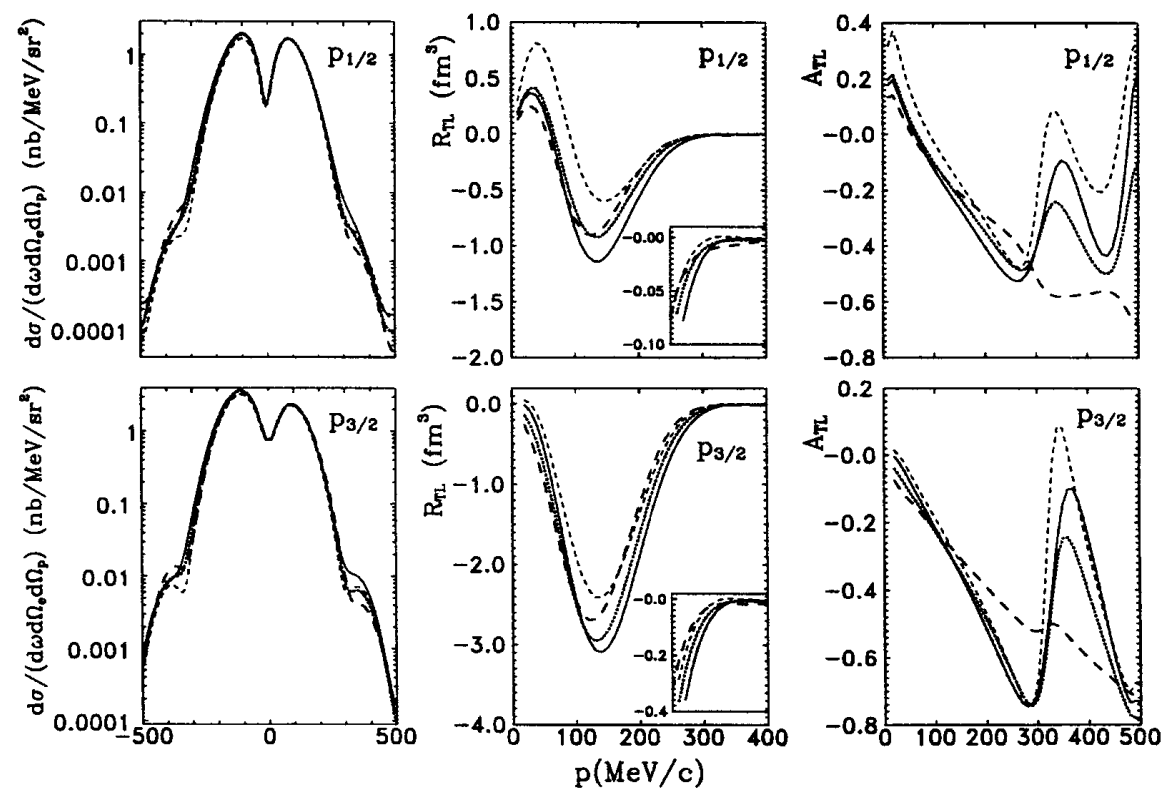

FIG. 1. Cross sections (left), $R_{\mathrm{TL}}$ (middle), and $A_{\mathrm{TL}}$ (right) for proton knockout from ${ }^{16} \mathrm{O}$ for the $1 p_{1 / 2}$ and $1 p_{3 / 2}$ orbits versus missing momentum $p_{m}$. Solid (dotted) line shows the fully relativistic calculation using the Coulomb gauge and the current operator $c c 1(c c 2)$. Also shown are the results after projecting the bound and scattered proton wave functions over positive-energy states $\left(J_{\text {proj }}\right.$, short-dashed line) and using the asymptotic momenta $\left(J_{\mathrm{as}}\right.$, long-dashed line). For the $p_{3 / 2}$ shell a small contribution from the nearby $5 / 2^{+}$and $1 / 2+$ states parametrized from the low- $p_{m}$ data [27] is included. Magnified plots of $R_{\mathrm{TL}}$ for $p_{m}>250 \mathrm{MeV} / c$ where accurate experimental data are being taken [3], are shown.

enhancement of the lower components is stronger for the $p_{1 / 2} R_{\mathrm{TL}}$ response than for $p_{3 / 2}$, a feature that was first seen in RPWIA [18] and that persists in this more realistic calculation including FSI. Particularly interesting is the oscillatory structure of the fully relativistic result for $A_{\mathrm{TL}}$. This characteristic is preserved in the $J_{\text {proj }}$ results, but not in the $J_{\text {as }}$ ones. The asymptotic projection severely modifies $A_{\mathrm{TL}}$ for both orbitals. We notice that the $A_{\mathrm{TL}}$ calculated with $J_{\text {as }}$ are very similar to the ones obtained in [24], particularly to the calculation called EMA(noSV) in said reference. At low momentum this approach is close to the fully relativistic and to the $J_{\text {proj }}$ results, but beyond $p_{m} \simeq 200 \mathrm{MeV} / c$ it gives different results. The oscillating trend of the $A_{\mathrm{TL}}$ calculated in RDWIA is confirmed by the preliminary data [3] and agrees qualitatively with previous calculations by Van Orden [3]. As it was the case for $R_{\mathrm{TL}}$, the negative energy components clearly affect $A_{\mathrm{TL}}$ for the $j=l-1 / 2$ partner, sizably driving the asymmetry towards negative values, even at low $p_{m}$.

Other relativistic effects can be seen in Fig. 2, where we compare RDWIA results on $A_{\mathrm{TL}}$ (left panels) and $R_{\mathrm{TL}}$ (right panels) to nonrelativistic approaches. We have used the $c c 2$ current operator and nonrelativistic scattered wave functions obtained from Dirac-equivalent Schrödinger equations [12]. Thus the nonrelativistic wave functions correspond to the upper components of the relativistic ones, containing the Darwin term. For the nonrelativistic bound wave functions, we used the ones in [28] where new approximations to the on-shell relativistic one-body current operator were developed to account better for relativistic kinematic effects in nonrelativistic calculations. In particular, the charge density contains a spin-orbit correction $\hat{\rho}_{\text {so }}$ that affects $R_{\mathrm{TL}}[28,29]$. In Fig. 2 we show by long dashed lines the results obtained with the "relativized current" and by dotted lines the results obtained when $\hat{\rho}_{\text {so }}$ is neglected. $\hat{\rho}_{\text {so }}$ has a large effect on $R_{\mathrm{TL}}$ and $A_{\mathrm{TL}}$. Using the DWEEPY [30] code we have obtained for $A_{\mathrm{TL}}$ similar results to the dotted lines in Fig. 2.

The short dashed lines in Fig. 2 are results obtained with the relativistic current and 4 -spinors constructed as in Eq. (2) from the nonrelativistic bound and scattered wave functions. In this way, all the elements of the relativistic calculation but the dynamical enhancement of the lower components are fully taken into account. This is why these results (short-dashed lines) for $A_{\mathrm{TL}}$ and $R_{\mathrm{TL}}$ are closer to the fully relativistic results (solid lines). Contrary to the effect of the dynamical enhancement of the lower components, the effects of relativistic kinematics are of the same order in both shells. The data on $A_{\mathrm{TL}}$ and $R_{\mathrm{TL}}$ are a strong indication of the presence and crucial role played by dynamical effects of relativity affecting the lower components, in electron-nucleus observables. Strong sensitivity of the TL response to relativistic corrections was earlier found for $d\left(e, e^{\prime} p\right)$ [31].

In conclusion, we have identified two types of relativistic effects on $R_{\mathrm{TL}}$ and $A_{\mathrm{TL}}$. One is of kinematical origin, and has a large contribution from the spin-orbit correction to the charge density, and the other is of dynamical origin. The latter is due to the enhancement of the lower components and is stronger for the $p_{1 / 2}$ than for the $p_{3 / 2}$ orbital. This is in addition to the dynamical effect on the upper 


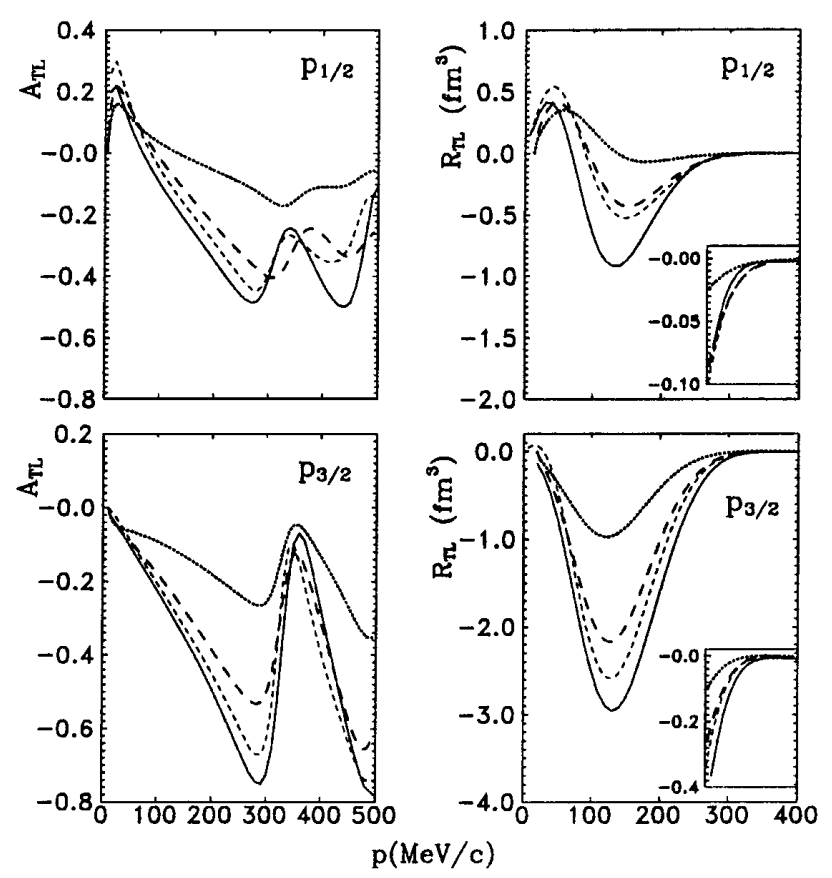

FIG. 2. $\quad R_{\mathrm{TL}}$ (right panels) and $A_{\mathrm{TL}}$ asymmetries (left panels) for proton knockout from ${ }^{16} \mathrm{O}$ for the $1 p_{1 / 2}$ (top panels) and $1 p_{3 / 2}$ (bottom panels) orbits. Results shown correspond to a fully relativistic calculation using the Coulomb gauge and the current operator $c c 2$ (solid line), a calculation performed by projecting the bound and scattered proton wave functions over positive-energy states (short-dashed line) and two nonrelativistic calculations with (long-dashed) and without (dotted) the spinorbit correction term in the charge density operator (see text for details).

component due to the Darwin term which is present in all the results given here, and that mostly affects the determination of spectroscopic factors [12]. It is encouraging that the preliminary data [3] agree so well with the predictions of the fully relativistic calculations, and one anticipates being able to make even more stringent tests when a finer grid of high-precision data involving other nuclei becomes available in the range $200 \leq p_{m} \leq 400 \mathrm{MeV} / c$.

We thank the TJNAF Hall A collaboration, especially W. Bertozzi, J. Gao, S. Gilad, and J.J. Kelly for their willingness to discuss their data prior to publication, and for many useful comments. This work was partially supported under Contracts No. 940183 (NATO Collaborative Research Grant), No. DE-FC01-94ER40818 (cooperative agreement with the U.S. Department of Energy DOE), No. PB/95-0123, No. PB/95-0533-A, No. PB/95-1204 (DG-ICYT, Spain), No. PB/96-0604 (DGES, Spain), No. PR156/97 (Complutense University, Spain), and by the Junta de Andalucía (Spain).

[1] S. Boffi, C. Giusti, and F. D. Pacati, Phys. Rep. 226, 1 (1993); S. Boffi, C. Giusti, F. Pacati, and M. Radici,
Electromagnetic Response of Atomic Nuclei (Clarendom Press, Oxford, 1996); J. J. Kelly, Adv. Nucl. Phys. 23, 77 (1996).

[2] L. Lapikas, Nucl. Phys. A553, 297c (1993); E. Quint, Ph.D. thesis, NIKHEF, 1988.

[3] T. Saha et al., TJNAF Proposal No. 89-003, 1989; J. Gao, Ph.D. thesis, MIT, 1999.

[4] J. Ryckebush, D. Debruyne, W. N. Nespen, and S. Janssen, nucl-th/9904011; V. Van der Sluys, J. Ryckebusch, and M. Waroquier, Phys. Rev. C 54, 1322 (1996).

[5] J. E. Amaro, A. M. Lallena, and J. A. Caballero, Phys. Rev. C 60, 014602 (1999).

[6] I. Bobeldijk et al., Phys. Rev. Lett. 73, 2684 (1994).

[7] V.R. Pandharipande, C. N. Papanicolas, and J. Wambach, Phys. Rev. Lett. 53, 1133 (1984); Z. Y. Ma and J. Wambach, Phys. Lett. B 256, 1 (1991); C. Mahaux and R. Sartor, Adv. Nucl. Phys. 20, 1 (1991).

[8] H. Müther, and W.H. Dickhoff, Phys. Rev. C 49, R17 (1994).

[9] L. Chinitz et al., Phys. Rev. Lett. 67, 568 (1991).

[10] A. Picklesimer and J. W. Van Orden, Phys. Rev. C 35, 266 (1987); 40, 290 (1989).

[11] J. M. Udías et al., Phys. Rev. C 48, 2731 (1993).

[12] J. M. Udías et al., Phys. Rev. C 51, 3246 (1995).

[13] J.P. McDermott, Phys. Rev. Lett. 65, 1991 (1990); Y. Jin, D. S. Onley, and L.E. Wright, Phys. Rev. C 45, 1311 (1992).

[14] J. M. Udías, P. Sarriguren, E. Moya de Guerra, and J. A. Caballero, Phys. Rev. C 51, R1488 (1996).

[15] T. de Forest, Nucl. Phys. A392, 232 (1983).

[16] B. D. Serot and J.D. Walecka, Adv. Nucl. Phys. 16, 1 (1986).

[17] E. D. Cooper, S. Hama, B. C. Clark, and R. L. Mercer, Phys. Rev. C 47, 297 (1993).

[18] J. A. Caballero, T. W. Donnelly, E. Moya de Guerra, and J. M. Udías, Nucl. Phys. A632, 323 (1998).

[19] J. A. Caballero, T. W. Donnelly, E. Moya de Guerra, and J. M. Udías, Nucl. Phys. A643, 189 (1998).

[20] S. Ulrych and H. Müther, Nucl. Phys. A641, 499 (1998).

[21] G. M. Spaltro et al., Phys. Rev. C 48, 2385 (1993).

[22] M. M. Sharma, M. A. Nagarajan, and P. Ring, Phys. Lett. B 312, 377 (1993).

[23] G. H. Rawitscher, Phys. Rev. C 31, 1173 (1985).

[24] J. J. Kelly, Phys. Rev. C 56, 2672 (1997); 60, 044609 (1999).

[25] S. Boffi, C. Giusti, F. D. Pacati, and F. Cannata, Nuovo Cimento 98, 291 (1987).

[26] Y. Jin and D. S. Onley, Phys. Rev. C 50, 377 (1994).

[27] M. Leuschner et al., Phys. Rev. C 49, 955 (1994).

[28] J.E. Amaro et al., Nucl. Phys. A602, 263 (1996); A611, 163 (1996).

[29] J. E. Amaro and T. W. Donnelly, Ann. Phys. 263, 56 (1998); Nucl. Phys. A646, 187 (1999); S. Jeschonnek and T. W. Donnelly, Phys. Rev. C 57, 2439 (1998).

[30] C. Giusti and F. Pacati, Nucl. Phys. A473, 717 (1987); A336, 427 (1980).

[31] S. Gilad, W. Bertozzi, and Z.-L. Zhou, Nucl. Phys. A631, 276c (1998); E. Hummel and J. A. Tjon, Phys. Rev. C 49, 21 (1994); J. E. Ducret et al., ibid. 49, 1783 (1994). 